\title{
Efficacy of Osteopathic Manipulative Treatment in the Patient of Chronic Rhinosinusitis: A Case Report
}

Nitish Baisakhiya

\begin{abstract}
Background: Chronic rhinosinusitis (CRS) is a most common chronic debilitating disease that has great economical impact and can affect different age-groups. It significantly affects the physical and mental domains of the patient, leading to the deterioration in the quality of life. Purpose: Since medical treatment is not yet standardized for CRS because of scarcity of knowledge about its etiology, and postsurgery relapses are common, the purpose of the present case study was to explore the efficacy of osteopathic manipulative treatment (OMT) in a CRS patient to prevent the relapse.

Case description: The author reported a 45-year-old female who presented with the complaints of facial pressure and pain, blocked nose, reduced sense of smell, pain around the eye, heaviness, ache in the head and neck on the right side with occasional postnasal drip and cough, unequal pressure in both the ears for the last couple of years, and always felt drain of energy. The patient had undergone pharmacological treatment for the same in the past, but her sinusitis persisted despite treatment. The X-ray (Water's view) of the paranasal sinus reveals opacification in the area of right maxillary sinus. The patient was treated with OMT technique twice a week for 4 weeks followed by the home exercise program. Results: The application of OMT revealed a significant change in the symptoms of the patient as self-reported and also improvement in the 22-item sinonasal questionnaire.

Conclusion: Osteopathic manipulative treatment could be used as a conservative treatment strategy in patients with chronic sinusitis. However, its effectiveness can only be explored in randomized clinical trials in order to generalize the results to a larger population.

Keywords: Manipulation, Manual therapy, Osteopathy, Rhinosinusitis, Sinusitis.

Clinical Rhinology An International Journal (2018): 10.5005/jp-journals-10013-1346
\end{abstract}

\section{INTRODUCTION}

Chronic rhinosinusitis (CRS) is a most common chronic debilitating disease with a prevalence of $10.6 \%$ in Europe and $16 \%$ in the United States. ${ }^{1,2}$ It has led to a great economic impact in terms of costeffectiveness and productivity. ${ }^{3}$ It is associated with the chronic inflammation of the nasal mucosa, persistent nasal blockage, facial pressure, and pain and chronic headaches, resulting in the negative impact on the quality of life (QoL) of sufferers., ${ }^{4,5}$ As the medical treatment is not yet standardized for CRS due to scarcity of knowledge about its etiology, postsurgery relapses are common. The conservative treatment in the form of physiotherapy (ultrasound therapy and low-level intraoral laser therapy) has been reported in the literature showing its benefits in patients with CRS. ${ }^{6,7}$ The underpinning of osteopathy is the convoluted relationship between the body framework and its function, along with self-healing capacity of the body from within. ${ }^{8}$ This concept can be utilized to treat patients with CRS. To the author's knowledge, there is scarcity of literature available that shows the efficacy of osteopathic manipulative treatment (OMT) in patients with CRS. The purpose of the present case study was to explore the efficacy of OMT in patients with CRS to prevent relapse. The QoL was assessed using subjective sinonasal questionnaire that is clinically valided ${ }^{9}$ into six grades, ranging from no problem to severe problem and consists of 22 items: need to blow nose, sneezing, runny nose, cough, post nasal discharge, thick nasal discharge, ear fullness, dizziness, ear pain, facial pain/pressure, difficulty falling asleep, waking up at night, lack of a good night's sleep, waking up tired, fatigue, low performance, reduced concentration, frustrated/ restless, sad, embarrassed, sense of taste/smell, and blockage/ congestion of nose.
Department of ENT, Maharishi Markandeshwar Institute of Medical Sciences and Research, Ambala, Haryana, India

Corresponding Author: Nitish Baisakhiya, Department of ENT, Maharishi Markandeshwar Institute of Medical Sciences and Research, Ambala, Haryana, India, Phone: +91 9588339184, e-mail: nitish. baisakhiya@gmail.com

How to cite this article: Baisakhiya N. Efficacy of Osteopathic Manipulative Treatment in the Patient of Chronic Rhinosinusitis: A Case Report. Clin Rhinol An Int J 2018;11 (2 and 3):58-60.

Source of support: Nil

Conflict of interest: None

\section{Case Description}

The author reported the case of a 45 -year-old female who selfreferred with chief complaint of facial pressure and pain, blocked nose, reduced sense of smell, pain around the eye, heaviness and ache in the head and neck on the right side with occasional postnasal drip and cough, unequal pressure in both the ears for the last couple of years, and she always felt drain of energy and disturbed sleep. The patient had no past medical and surgical history. The patient had undergone pharmacological treatment for the same in the past, but her sinusitis persisted despite treatment.

Physical examination suggested mild puffiness of the face on the right side in the area of nasolabial fold. The palpation revealed tenderness near the infra-orbital foramen. The nature of facial pain was dull aching in the right periorbital region, in the upper teeth, and, sometimes, in the right temple region. The osteopathic examination revealed restricted cranial movement with the right lateral strain at the basisphenoid junction, right temporal bone

() The Author(s). 2018Open Access This article is distributed under the terms of the Creative Commons Attribution 4.0 International License (https://creativecommons. org/licenses/by-nc/4.0/), which permits unrestricted use, distribution, and non-commercial reproduction in any medium, provided you give appropriate credit to the original author(s) and the source, provide a link to the Creative Commons license, and indicate if changes were made. The Creative Commons Public Domain Dedication waiver (http://creativecommons.org/publicdomain/zero/1.0/) applies to the data made available in this article, unless otherwise stated. 


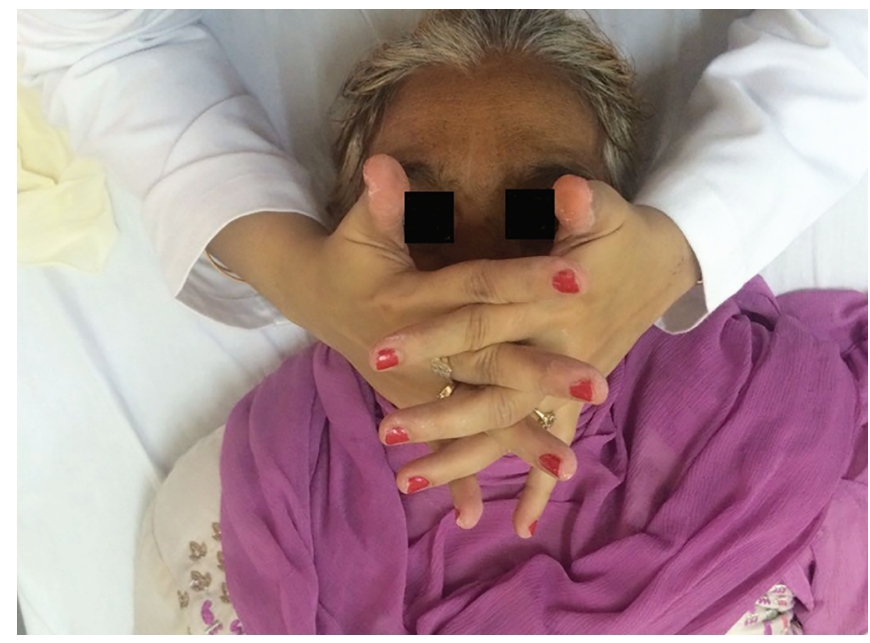

Fig. 1: Counterstrain maxillary tender point

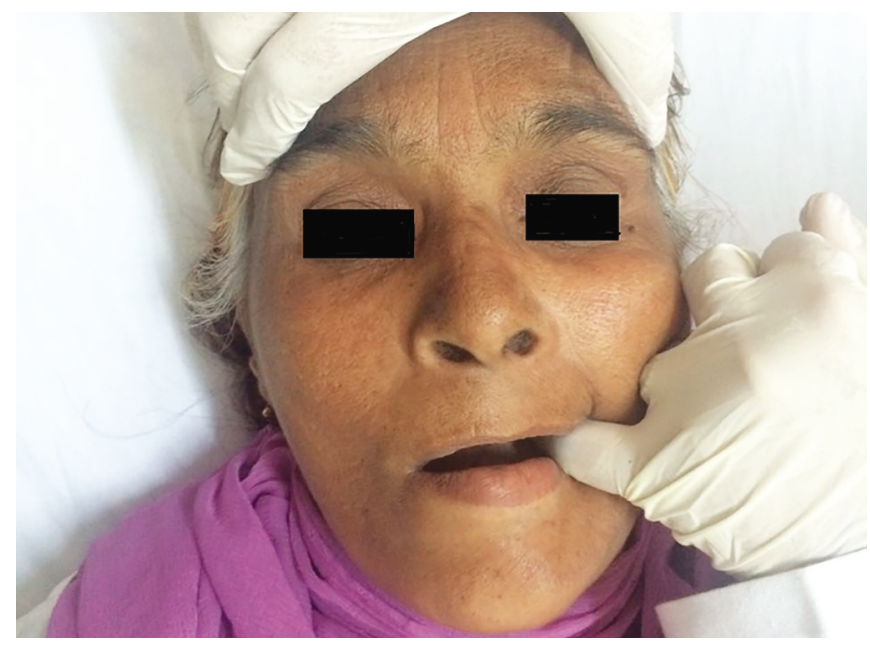

Fig. 3: Intraoral sphenopalatine ganglion decongestion technique

restricted in external rotation, the cranial rhythmic impulse was reduced, and the motion at the base of the sacrum and occipitalatlantal $(\mathrm{OA})$ region was restricted. Intraoral examination revealed normal dentition and mucosal lining except for flattened roof of the mouth, which signifies that vomer was flexed, and during palpation, the pain was elicited on the right side of the mouth and the maxillopalatine suture restriction was felt, which signifies the torsional deviation of vomer to right. Palpatory examination also showed restricted movement, tenderness, tissue texture changes, and asymmetry at all the major diaphragms', namely, pelvic diaphragm, abdominal diaphragm, thoracic inlet, and hyoid diaphragms', respectively, mostly on the right side defining the somatic dysfunction.

The Water's view showed opacification in the area of right maxillary sinus and also the preserved but blurred sinus walls. Based on the clinical symptoms and the radiographic projection, the diagnosis of chronic maxillary rhinosinusitis was made per the diagnostic criterion. ${ }^{10}$

The osteopathic manipulative techniques were chosen in order to address the symptoms of the patient. The techniques applied were myofascial release of all major diaphragms in order to release the restrictions. The dural tube rocking was performed in order to improve the amplitude of the cranial rhythmic impulse. Generalized

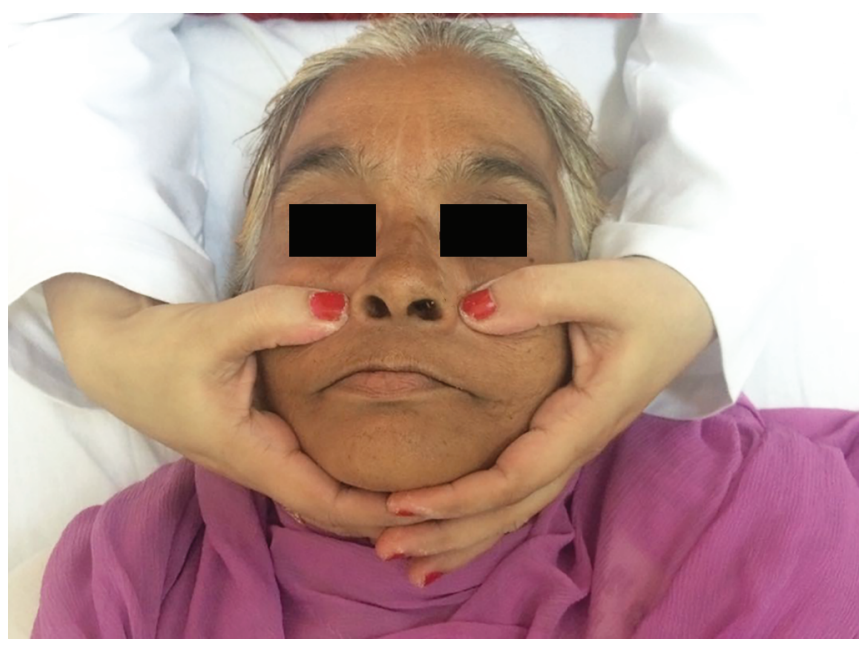

Fig. 2: Milking technique of maxillary sinus

sphenoidal release to address right lateral strain, mastoid wobble technique to improve temporal bone movement, OA release technique to treat somatic dysfunction, and intraoral vomer and maxillopalatine manipulation were performed to reduce the dural tension and hence to improve the cranial rhythm. The counterstrain maxillary sinus technique was performed in order to relieve the tender point near the nasolabial fold. The "milking technique" to the maxillary sinus for 6 cycles with 3 minutes each was performed in order to improve the lymphatic blockage (Figs 1 and 2). The sphenopalatine ganglion decongestion technique was also performed in order to improve the blocked nose. The patient was suggested to do deep breathing exercises at home (10 times/3 sets) along with steam inhalation for 15 minutes at bed time (Fig. 3).

The treatment was given twice a week for 4 weeks, and the patient self-reported significant improvement in all the symptoms, and the 22-item sinonasal questionnaire showed significant improvement in the items.

\section{Discussion}

The purpose of the present case study was to explore the efficacy of osteopathic treatment (OT) in CRS-induced disabilities in the patient. The osteopathic philosophy states that the structure and function within the human body is interrelated. The osteopathy approach provokes the internal mechanisms of the body to heal itself by the use of human hands. ${ }^{11}$ The OT centers on the nervous system, the circulatory system, the spine, the viscera, and the thoracic and pelvic diaphragms.

"Sinusitis is almost always in conjunction with somatic dysfunction of the upper cervical spine. Sympathetic innervation to the sinus areas arises from the upper thorax and travels through the cervical region. An OA somatic dysfunction is the most common. Treating the cervical somatic dysfunctions and performing sinus drainage techniques help to relieve pain as well as assist in drainage of the sinuses." ${ }^{12}$

Milking technique was used to improve the lymphatic drainage, which augments the removal of metabolic wastes from the mucosa and also improves the transportation of the nutrients and hence maintains the internal homeostasis. The sphenopalatine ganglion is the parasympathetic secretomotor supply to the nasal glands, and its activation will stimulate the parasympathetic nervous system that helps in the clearance of the secretions from the mucosa and sinuses. $^{12}$ 
The maxillary counterstrain technique reduced the hypertonicity of the muscle by placing it in the shortened position or the position of comfort, which improves the gamma gain of the stretch reflex resulting in the reduced pain and increase in the range of motion as proposed by Jones. ${ }^{13}$

Cranial osteopathy techniques-mastoid wobble, dural tube rocking, generalized sphenoidal release, and vomer and maxillopalatine suture manipulation-performed in the present case study helps in restoring the primary respiratory mechanism by reducing the dural tension and maintaining the cranial rhythmic impulse (Figs 1 to 3 ).

The efficacy of OMT techniques in sinusitis was explored by Lee-Wong et al. ${ }^{14}$ and concluded that it may be beneficial in the treatment of chronic sinusitis. The results procured in the present case study are bolstered by the study conducted by Lee-Wong et al.

However, the results obtained cannot be generalized to the larger population. The future randomized clinical trials with the larger sample size should be conducted to prove the effectiveness of OMT in CRS. Also, the objective outcome measure should also be included to note the change in the symptoms of the patient.

\section{Conclusion}

The OMT application in the patients with CRS significantly improved the clinical symptoms on the SNOT 22 questionnaire. Therefore, it may be use a conservative treatment choice in CRS because of no side effects.

\section{References}

1. Hastan D, Fokkens WJ, Bachert C, et al. Chronic rhinosinusitis in Europe-an underestimated disease. A GA (2) LEN study. Allergy 2011;66(9):1216-1223. DOI: 10.1111/j.1398-9995.2011.02646.x.

2. Pleis JR, Coles R. Summary health statistics for U.S. Adults: national health interview survey, 1998. vital health. Stat 2002;10(209):1-113.
3. Halawi AM, Smith SS, Chandra RK. Chronic rhinosinusitis: epidemiology and cost. Allergy Asthma Proc 2013;34(4):328-334. DOI: 10.2500/aap.2013.34.3675.

4. Fu Q-L, Ma J-X, Ou C-Q, et al. Influence of self-reported chronic rhinosinusitis on health-related quality of life: a population-based survey. PLoS ONE 2015;10(5):e0126881. DOI: 10.1371/journal. pone.0126881.

5. Prateek S, Banerjee G, Gupta P, et al. Fungal rhinosinusitis: a prospective study in a university hospital of Uttar Pradesh. Indian J Med Microbiol 2013;31(3):266-269. DOI: 10.4103/0255-0857.115634.

6. Ansari NN, Fathali M, Naghdi S, et al. A randomized, double-blind clinical trial comparing the effects of continuous and pulsed ultrasound in patients with chronic rhinosinusitis. Physiother Theory Pract 2011;28(2):85-94. DOI: 10.3109/09593985.2011.571751.

7. Mortazavi $H$, Khalighi $H$, Goljanian A, et al. Intra-oral low level laser therapy in chronic maxillary sinusitis: a new and effective recommended technique. J Clin Exp Dent 2015;7(5):e557-e562. DOI: 10.4317/jced.52282.

8. Ward RC. Foundations for Osteopathic Medicine. 2nd ed., New York: Lippincott Williams \& Wilkins; 2003.

9. Hopkins C, Gillett S, Slack R, et al. Psychometric validity of 22-item sinonasal outcome test. Clin Otolaryngol 2009;34(5):447-454. DOI: 10.1111/j.1749-4486.2009.01995.x.

10. Rosenfeld RM, Piccirillo JF, Chandrasekhar SS, et al. Clinical practice guideline: Adult sinusitis. Otolaryngol Head Neck Surg 2015;152(S2):s1-s39. DOI: 10.1177/0194599815572097.

11. Branyon B. Healing hands: using osteopathic manipulative treatment to address visceral structures through somatovisceral reflexes: a case study in gastroesophageal reflux disease. AAO Journal 2008;18(4): 29-31.

12. DiGiovana E. An Osteopathic Approach to Diagnosis and Treatment. 3rd ed., Philadelphia: Lioppincott Willaims \& Wilkins; 2005.

13. Jones LH, Kusunose RS, Goering EK. Strain-Counterstrain. Boise, Idaho: Jones Strain-Counterstrain Inc; 1995. DOI: 10.1016/0264-410x(95)00019-w.

14. Lee-Wong M, Karagic M, Doshi A, et al. An osteopathic approach to chronic sinusitis. J Aller Ther 2011;2(2):109. DOI: 10.4172/21556121.1000109. 